\title{
An Optimum Source Coverage Adaptive Gateway Discovery for Heterogeneous Networks
}

\author{
Rashmi Kushwah $^{1}$, Shashikala Tapaswi ${ }^{1}$, and Ajay Kumar ${ }^{1}$ \\ ${ }^{1} A B V$-Indian Institute of Information Technology and Management, \\ Gwalior, India \\ \{rashmik, stapaswi,ajayfma\}@iiitm.ac.in
}

\begin{abstract}
A Mobile ad hoc network (MANET) is a standalone network in which the communication of mobile nodes (MNs) is restricted within its own premises. To enhance the communication range of mobile nodes, MANET is connected to the Internet using Internet Gateways (IGs). Since IG is used to integrate MANET with the Internet, efficient selection of IG is important for mobile nodes to discover the routes to the IG. This paper aims to develop an adaptive IG discovery approach that dynamically modifies the proactive area. The proposed scheme considers the average number of hop count and standard deviation of hop count of all the Active Sources (ASs) which desire Internet connectivity. Simulations results show that proposed scheme based on optimum source coverage perform better than existing IG discovery mechanism.
\end{abstract}

Keywords: MANET, Internet gateway, Mobile IP, Adaptive gateway discovery

\section{Introduction}

MANET [1] is a self-configurable wireless network consists of MNs. MANET is suitable in those situations where temporary communication is required. To enhance the coverage of mobile nodes MANET is connected to the fixed network and thus forms a hybrid MANET. The interconnection is achieved through some special nodes called gateway nodes which consist of both wired and wireless interface. When MANET is connected to the Internet, mobile node should select a nearby IG. Internet connectivity is supported by three types of discovery scheme for IG that are proactive, reactive and hybrid [2]. In proactive scheme, the IG broadcast an Internet Gateway Advertisement (IGADV) message in a fixed interval of time throughout the MANET. This approach provides good connectivity by broadcasting IGADV frequently at the cost of high control overhead. In reactive scheme, AS sends Internet Gateway Solicitation (IGSOL) message to discover the IG. When IG receives the message, it replies back to the AS. This can be done with less overhead but incurs more delay. Hybrid scheme combines the advantages of both the proactive and reactive scheme [2]. After discovering multiple routes to the IG, AS selects best IG to communicate with the Internet. In last few years, a lot of research has been performed on MANET routing protocols [3] and a little attention has been given to adaptive gateway discovery for MANET and Internet connectivity. It is assumed that one or more IGs are present to connect MANET to the Internet. Existing IG discovery mechanism such as proactive, reactive and hybrid are only suitable for certain network configuration and not adaptable to dynamic network conditions. Scalability problems arise when proactive area does not reflect the dynamic configuration of MANET. Therefore a fixed Time to Live (TTL) value is not a good choice for dynamic network condition in MANET.

Earlier studies were focused on maximum or average value of hop count of ASs for adjusting the proactive area that needs Internet connectivity. The major drawbacks of these schemes were that they suffer from large overhead and thereby lead to inefficient 
utilization of bandwidth and resources. The aim of proposed scheme is to design an adaptive IG discovery mechanism to get the optimal performance which dynamically changes the proactive area using the average number of hop count and standard deviation of hop count of all the Active Sources (ASs) which desire Internet connectivity. This results in reduction of IG latency in such a way that more ASs will be covered.

The rest of the paper is organized as follows: Section 2 presents the related work. Section 3 discusses the proposed adaptive gateway discovery approach. Section 4 evaluates and compares the gateway discovery mechanism by simulation. Section 5 concludes the paper.

\section{Related Work}

When MN sends information toward the fixed host, it should discover an IG. An active source should be able to select one of the IGs among the multiple IGs and to support the mobility of MANET node, Mobile IP [4] is used with the MANET. A global address is required by the AS to communicate with the fixed host when MANET is connected to an IP network. Some of the IG discovery proposals are as follows:

In [5] a maximal source coverage algorithm is proposed in which the TTL value of next IGADV is determined by the maximum number of hops among all its ASs. The above scheme is not applicable in all situations since different part of the network may have different load condition. In [6] a new scheme is proposed in which IGADV is targeted only at ASs and not at other MNs. In this way only ASs receive the IGADV message and other MNs are not loaded. In [7] a maximal benefit coverage algorithm is proposed in which the TTL value of IGADV is based on maximal source coverage algorithm and the adjustment of periodicity of IGADV is adjusted based on mobility of MANET nodes. [8] presents an extension of routing mechanism originally employed in Tree or Linear Hopping network (ToLHnet protocol) [9]. This aimed to provide better handling of linear routing over a wired link such as an RS-485 bus. With this extension it is possible to reduce latency and increase throughput for short distance communication. [10] presents an enhancement of Modbus RTU (Remote Terminal Unit) by enabling a wired and wireless tree bus topology and master multiplexing techniques.

\section{Proposed Adaptive Gateway Discovery Approach}

\subsection{Network Model}

The network model of hybrid MANET is shown in Fig 1. A MANET can be formulated as an infrastructure less network which can be represented by a graph $G(V$, $\mathrm{E}) . \mathrm{V}=\left\{\mathrm{v}_{1}, \mathrm{v}_{2} \ldots \mathrm{v}_{\mathrm{n}}\right\}$ is the set of $\mathrm{n}$ mobile nodes representing MNs and IGs. $\mathrm{E}$ is the set of communication links between two MNs. $Z=\left\{z_{1}, z_{2} \ldots z_{m}\right\}$ is the set of ASs such that $m \leq n$ and $\mathrm{Z} \subseteq \mathrm{V}$. It is assumed that all the ASs are normally distributed which means that most of the ASs are close to the average number of hop count of all AS while relatively few ASs tend to one extreme or the other. Large standard deviation leads to wider and lower curves whereas smaller standard deviation leads to narrower and taller curves. It is assumed that maximum concentration of ASs lies within the standard deviation from the mean value. Same principle is used in the proposed optimum source coverage algorithm. 


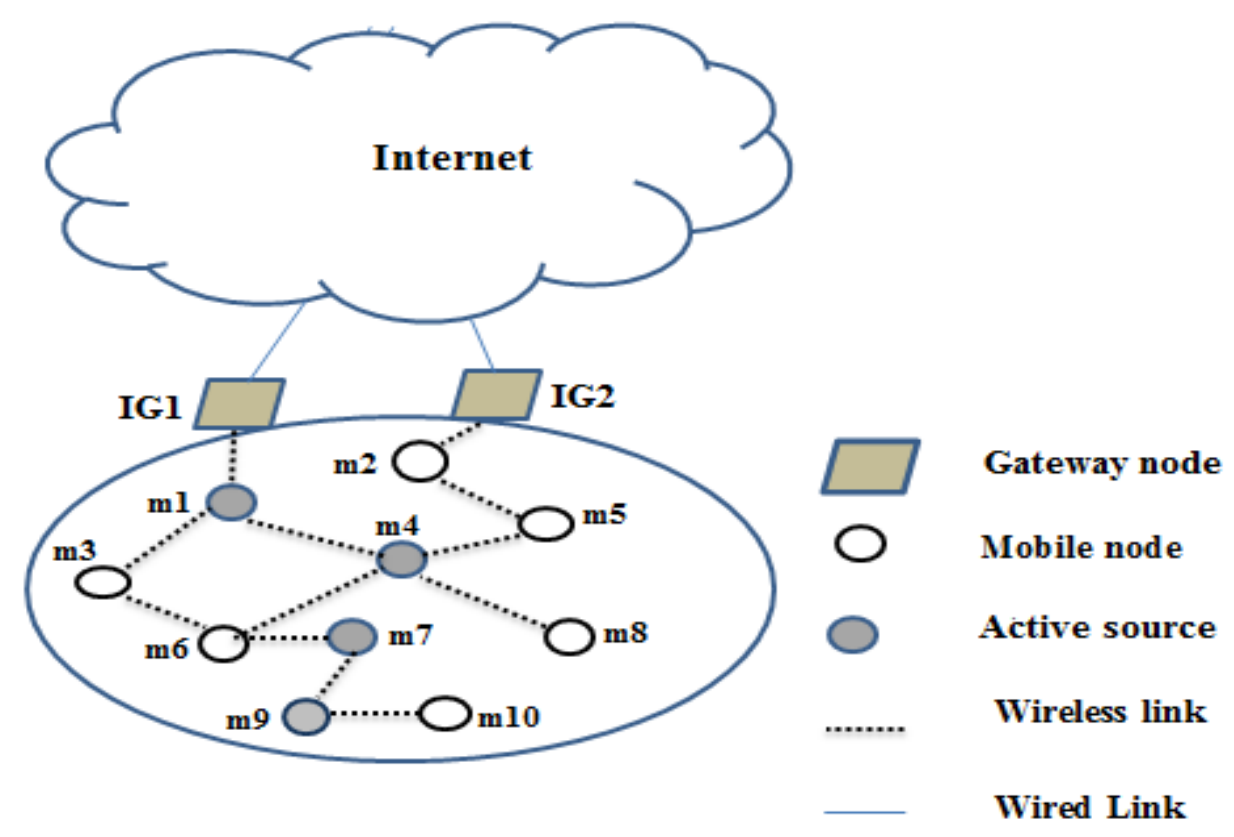

Figure 1. Adaptive IG Discovery in Hybrid MANET

\subsection{Computing Proactive Area}

IGs periodically announce their presence by broadcasting IGADV message in the MANET. To avoid flooding in the MANET, the IGADV messages are restricted within $n$ hop using TTL field. This range is called proactive range which determines the IG discovery scope that is dynamically adjusted by proposed approach. The proposed adaptive IG discovery approach states that the proactive area is dynamically adjusted based on the average number of hops of all ASs and standard deviation of hop count of all ASs to quantify the amount of variation of a set of hop count of the ASs. The maximum source coverage algorithm [5] which considers the farthest distance of AS for next TTL is not suitable to all situations where most of the ASs are near the average of hop count and very few are far from the gateway. In this case, maximum source coverage algorithm is not efficient since it generate a large amount of overhead. Simultaneously if we consider the average source coverage algorithm suggested by [5] which consider the average hop count of all Ass is also not applicable in those situations where most of the ASs are far from the gateway and a very few near to the gateway, since this algorithm does not cover most of the ASs which are outside their TTL range. To overcome the limitation of both the above approaches an adaptive gateway discovery approach is proposed in which the TTL for the next IGADV is set between the farthest AS and average of all ASs. To define the proactive range, IGs need the information of all the ASs present in the MANET. The AS is the MN which is connected with current IG and sends the data packet to the Internet through this IG. The IGs accumulate the traffic of all ASs in a multihop fashion which in turns sends to the fixed network.

The two terms which are used to define TTL of next IGADV are defined as follows:

1) The average number of hops of all ASs (Avghop_ASs), calculates the average number of hops of all its ASs. It can be represented as:

$$
\text { Avghop_ASs }=\sum_{\mathrm{i}=1}^{\mathrm{m}} \mathrm{z}_{\mathrm{i}} / \mathrm{m},
$$

2) The standard deviation of the number of hops of Ass (stdevhop_ASs), calculates the standard deviation of the number of hops of all ASs. It is the most widely used 
measure of dispersion and defines the variation of ASs from the mean. It can be calculated as:

$$
\text { Stdevhop_ASs }=\sqrt{\left(\sum_{i=1}^{m}\left(z_{i}-z^{r}\right)^{2}\right) / m},
$$

The TTL value of next GWADV is calculated as:

$$
\begin{aligned}
\mathrm{TTL}_{\text {next }} & =\text { Avghop_ASs }+ \text { Stdevhop_ASs, } \\
\mathrm{TTL}_{n e x t} & =\sum_{\mathrm{i}=1}^{\mathrm{m}} \mathrm{z}_{\mathrm{i}} / \mathrm{m}+\sqrt{\left(\sum_{\mathrm{i}=1}^{\mathrm{m}}\left(\mathrm{z}_{\mathrm{i}}-z^{s}\right)^{2}\right) / m},
\end{aligned}
$$

where $m$ is the total number of ASs and $z$ ' is the average number of hop count of all ASs. Using standard deviation, the proactive area is dynamically adjusted according to the TTLnext which is set in the IGADV message by IGs during next time interval. It will increase the proactive area so that more ASs can send data packet toward the fixed host. The low value of standard deviation indicates that the ASs tend to be close to the mean value while a high value indicates that the ASs are spread out from the mean. By adding the value of standard deviation to the average number of hop count of all its ASs will dynamically change the proactive area and provide wider coverage with less overhead. The resulting proactive range is smaller than the maximum source coverage algorithm while greater than the average source coverage algorithm as shown in Fig 2(c). The proactive range of maximum and average source coverage algorithm is shown in Fig 2(a) and 2(b) respectively.
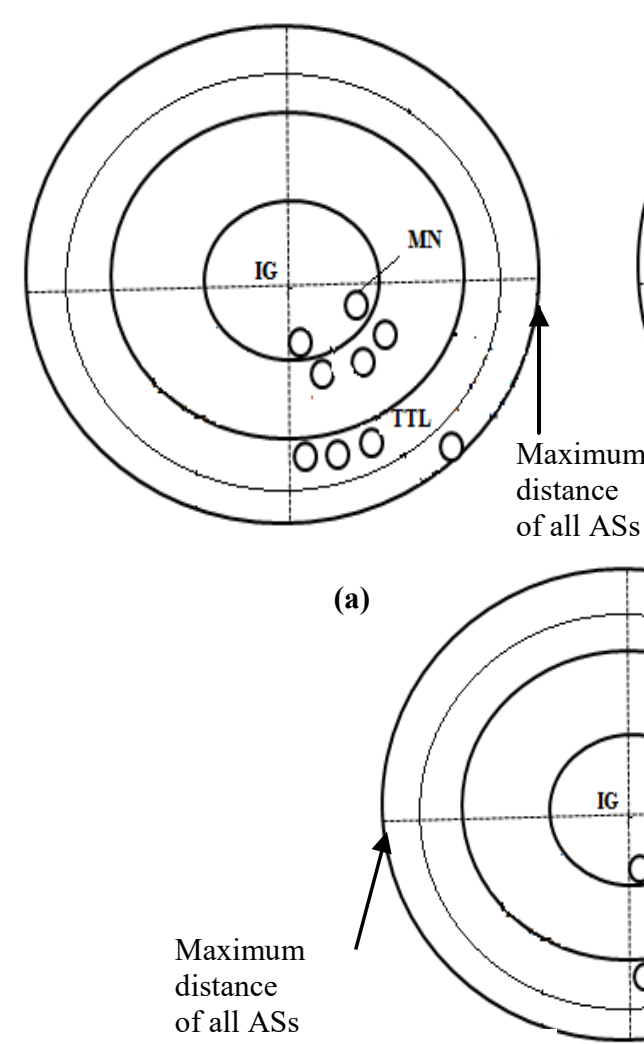

(a)

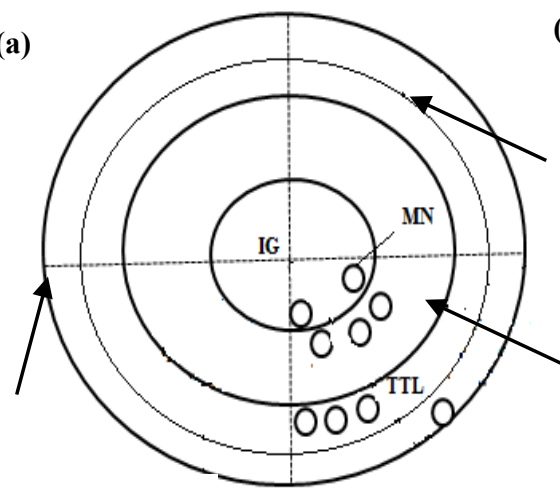

(c)

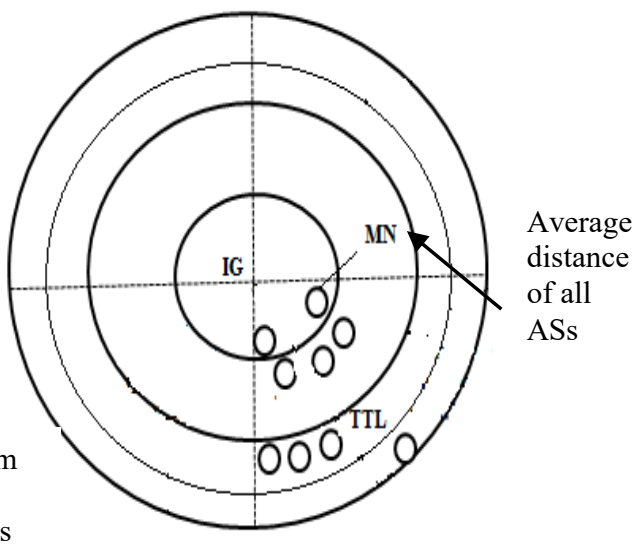

(b)

Proactive area created by proposed approach

Average distance of all ASs

Figure 2. (a) Maximum Source Coverage Algorithm Set TTL According to Farthest ASs. (b) Average Source Coverage Algorithm Set TTL to Average Distance of all ASs. (c) Proposed Approach set TTL between farthest AS and Average Distance of all ASs which Cover Maximum Node as Possible 


\subsection{Adaptive IG Discovery Algorithm}

The pseudocode of proposed adaptive IG discovery algorithm is given in Algorithm 1. In step 1 IG obtains hop count of all ASs from IP header of their data packets. Average and standard deviation of the number of hop count of all ASs is calculated in step 2 and 3 respectively. Step 4 calculates the TTL value of next IGADV. Further, IGADV is broadcasted up to the value of TTLnext.

Algorithm 1: Adaptive IG discovery algorithm

1: IG obtains hop count information from IP header of data packet sent by ASs;

2: IG calculates average number of hop count of all ASs;

Avghop_ASs $=\sum_{\mathrm{i}=1}^{\mathrm{m}} \mathrm{z}_{\mathrm{i}} / \mathrm{m}$

3: IG calculates standard deviation of hop count of all the ASs;

Stdevhop_ASs $=\sqrt{\left(\sum_{i=1}^{m}\left(z_{i}-z^{s}\right)^{2}\right) / m}$

4: TTL for next IGADV is calculated as;

$$
\mathrm{TTL}_{\text {next }}=\sum_{\mathrm{i}=1}^{\mathrm{m}} \mathrm{z}_{\mathrm{i}} / \mathrm{m}+\sqrt{\left(\sum_{\mathrm{i}=1}^{\mathrm{m}}\left(\mathrm{z}_{\mathrm{i}}-z^{\prime}\right)^{2}\right) / m}
$$

5: IG sends (IGADV, TTL next $_{\text {) }}$ to the immediate neighbor ASs to the initial

Value TTL=1, Set TTL=TTL+1;

7: IGADV is broadcast up to the TTL $\mathrm{TLx}_{\text {nex }}$;

8: End procedure

\subsection{IG Discovery Process}

As shown in Figure 1 there are 4 ASs which are $\mathrm{m} 1, \mathrm{~m} 4, \mathrm{~m} 7$ and $\mathrm{m} 9$ present in the network with their hop distance 1, 2, 4 and 5 respectively from the IG. If maximum source coverage algorithm is used the TTL of next IGADV is 5 , the farthest distance of AS from the IG which covers all ASs but with large overhead. However, if average source coverage algorithm is considered then only node $\mathrm{m} 1$ and $\mathrm{m} 4$ comes within proactive range and $\mathrm{m} 7$ and $\mathrm{m} 9$ have to discover the IG reactively because the average hop count of four ASs is 3 which is set as a TTL of next IGADV message. With proposed approach, the TTL of next IGADV message is set to 4 . The proposed algorithm cover $75 \%$ of ASs with less overhead and end to end delay as compared to maximum source coverage algorithm. In this way, the TTL of next IGADV is dynamically adjusted, and $\mathrm{m} 7$ comes within the proactive range, and there is no need for it to discover the IG reactively. IG obtains the information of the number of hop count of ASs which is required for adaptive IG discovery. The IP header of the data packet sent by AS contains the required information as shown in Table 1. 
Table 1. Hop Count Information Maintained by IG1

\begin{tabular}{ccc}
\hline S.No & IP address & Hop count \\
\hline 1 & $192.168 .100 .1\left(\mathrm{~m}_{1}\right)$ & 1 \\
2 & $192.168 .100 .2\left(\mathrm{~m}_{4}\right)$ & 2 \\
3 & $192.168 .100 .3\left(\mathrm{~m}_{7}\right)$ & 4 \\
4 & $192.168 .100 .4\left(\mathrm{~m}_{9}\right)$ & 5 \\
\hline
\end{tabular}

\section{Simulation Results}

To analyze the performance of proposed scheme Opnet modeler 18.0 is used. There are 25 mobile nodes distributed randomly in the area of $1000 \times 1000 \mathrm{~m}$. Two IGs are located at the coordinate $(65.61,35.57),(69.37,451.30)$, with $\mathrm{TTL}=2$ for their advertisement. The communication range of $\mathrm{MNs}$ is $300 \mathrm{~m}$, the MNs communicate with each other via 802.11b DCF MAC layer, with wireless channel capacity for each MN is $2 \mathrm{Mb} / \mathrm{s}$. Additionally, each router connected to a fixed host with a fixed link having the bandwidth $10 \mathrm{Mb} / \mathrm{s}$ which is sufficient to handle all the traffic from the MNs. Each MN uses a random waypoint mobility model with a speed of $15 \mathrm{~m} / \mathrm{sec}$. Each of the source nodes generates data packets of 512 bytes at a rate of 4 packet/sec. Two different traffic loads are tested with 5 and $10 \mathrm{CBR}$ source nodes communicating with fixed node on the Internet. The value of various simulation parameters used is shown in Table 2.

Following performance metrics have been used to analyze the effect of proposed approach.

1) Throughput: - It is the ratio of the total packet received successfully by the IG to the packet sent by the AS.

2) End to End (ETE) delay: - It is the average delay for the packet to send successfully from AS to IG.

\section{Table 2. Simulation Parameters}

\begin{tabular}{ll}
\hline \multicolumn{1}{c}{ Simulation Parameter } & \multicolumn{1}{c}{ Value } \\
\hline Simulation area & $1000 \times 1000 \mathrm{~m}$ \\
Simulation duration & $600 \mathrm{sec}$ \\
Wireless MAC interface & $802.11 \mathrm{~b}$ \\
Network Size & 25 mobile node \\
Number of IG & 2 \\
Number of source node & 5,10 \\
Packet size & $512 \mathrm{byte}$ \\
Radio range of mobile node & $300 \mathrm{~m}$ \\
Number of fixed node & 4 \\
Mobility pattern of node & Random Way point \\
Node speed & $15 \mathrm{~m} / \mathrm{Sec}$ \\
pause time period & $20 \mathrm{~s}$ \\
Traffic Source & $\mathrm{CBR}$ \\
&
\end{tabular}

\subsection{Results and Analysis}

To compare the proposed adaptive IG discovery scheme with existing IG discovery approaches such as maximum source coverage algorithm, reactive and hybrid approaches, 
a set of simulation runs have been performed by setting the static value of TTL $=2$ in terms of ETE delay and average throughput with 5 and 10 ASs.

Figure 3(a) shows that the proposed approach with 5 ASs is able to offer higher throughput than reactive and hybrid approaches and almost the same throughput than the maximum source coverage algorithm. The reason is that in hybrid approach the fixed TTL value is used so IGADV message could reach to the limited ASs. While in proposed scheme the TTL for every IGADV is dynamic and can use higher TTL so that more ASs can listen to the IGADV message and hence throughput of proposed adaptive scheme increases. Similarly, in the reactive scheme the mobile node discovers the IG reactively which incurs less throughput.

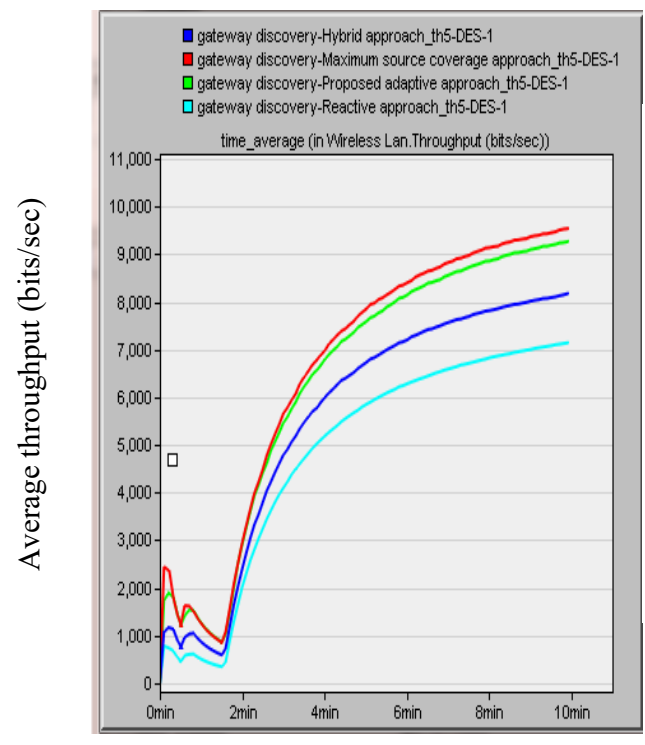

Time (minutes)

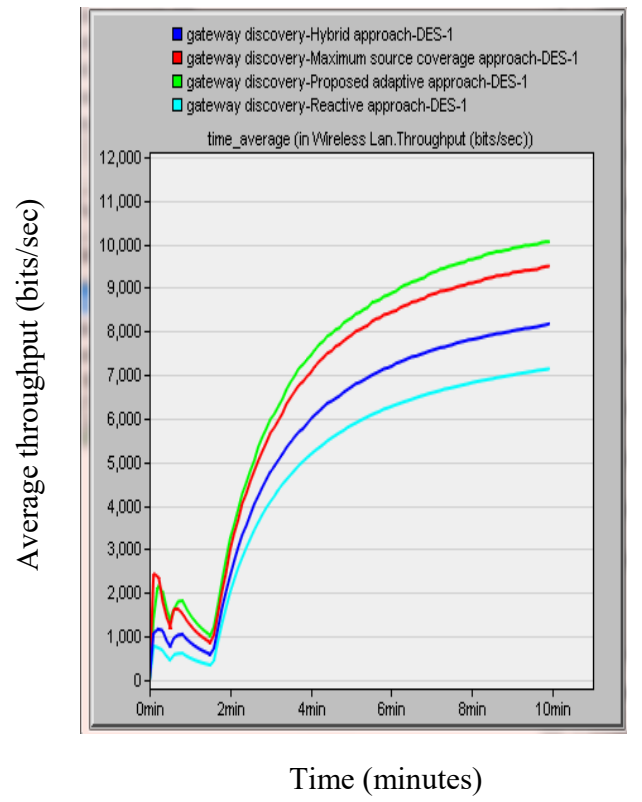

(b)

(a)

Figure. 3. Comparison of proposed Adaptive Scheme Vs Existing Mechanism for Average Throughput (a) with 5 Ass (b) with 10 ASs

Figure 3 (b) shows that the proposed scheme with 10 ASs provides higher throughput when compared to existing IG discovery mechanism. The hybrid and reactive approaches require large overhead due to more number of ASs needs to discover the IG reactively, which degrades the throughput of the network. The maximum source coverage algorithm gives poor performance due to high number of control packets exchange, consumes all the resources of the network. However, in proposed scheme, the TTL is dynamically adaptable and use an optimal value of TTL so that almost all ASs can get IGADV packet from IGs with less overhead and there is no need to discover the IG reactively. 


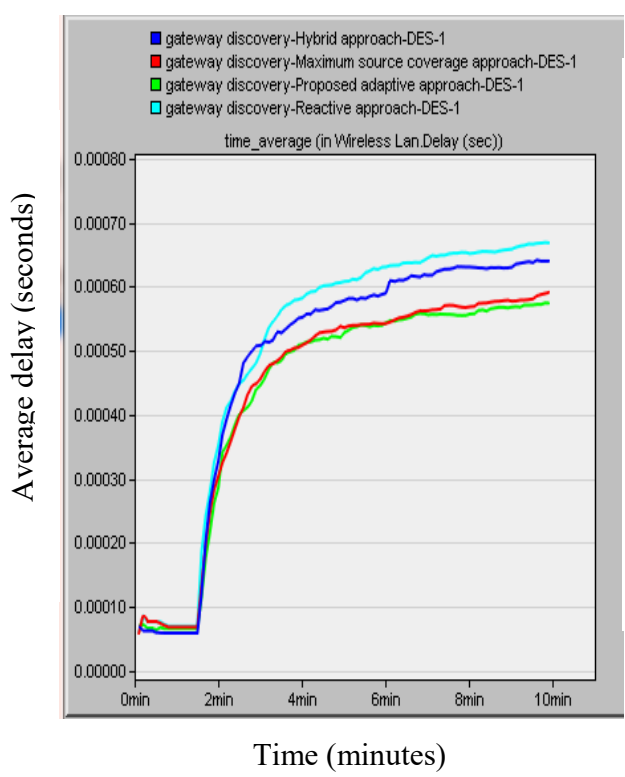

(a)

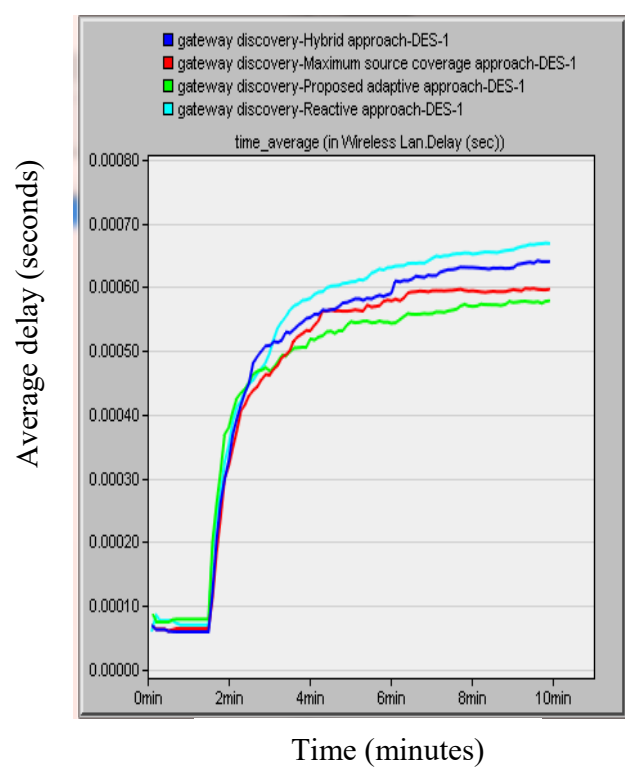

(b)

\section{Figure 4. Comparison of Proposed Adaptive Scheme vs. Existing Mechanism for Average ETE Delay (a) with 5 Ass (b) with 10 ASs}

As shown in Figure 4 (a) the proposed adaptive IG discovery approach with 5 active sources has lower ETE delay than reactive and hybrid approaches and almost equal to the maximum source coverage algorithm. With the minimum number of ASs, the proposed scheme and maximum source coverage algorithm have nearly equal coverage which leads to almost equal ETE delay. Figure 4 (b) shows that the proposed adaptive IG discovery approach with 10 ASs has lower ETE delay than reactive, hybrid and maximum source coverage schemes. In the proposed adaptive IG discovery approach, the TTL is dynamically adjusted based on average and standard deviation of the hop count of all the ASs that reduces excessive traffic and requires minimum time to discover the routes by confining the IGADV in a limited area resulting the shorter path length and smaller ETE delay.

While comparing the simulation results in terms of throughput it is observed that, the higher the number of ASs, the proposed approach gives maximum throughput as compared to existing schemes [5, 2]. The proposed approach gives almost equal throughput with less ASs as compared to the maximum source coverage algorithm due to the fact that it uses an adaptive TTL value which covers all the ASs with less overhead that leads to almost equal throughput. The deviation in terms of ETE delay is also minimum as the number of ASs increases in the network.

\section{Conclusion}

In this paper, an adaptive IG discovery approach has been proposed that consider the average number of hop count and standard deviation of hop count of all ASs which desires Internet connectivity. By using standard deviation, proposed approach adjusts the TTL dynamically between farthest ASs and the average distance of all ASs which leads to wider coverage of ASs, this results into more ASs to send data packets towards the fixed network, and increase the throughput of the network. The current IG discovery approaches: reactive, hybrid and maximum source coverage have been discussed and compared with the proposed scheme. Simulation results show that the proposed approach has less delay and good connectivity as compared to other existing mechanism. 


\section{Acknowledgments}

The authors would like to thank ABV-Indian Institute of Information Technology and Management, Gwalior, India for providing the infrastructure and academic support.

\section{References}

[1] M. Conti and S. Giordano, "Mobile ad hoc networking: Milestones, challenges, and new research directions", IEEE Communications Magazine, vol. 52, no. 1, (2014), pp. 85-96.

[2] R. Attia, R. Rizk and H. A. Ali, "Internet connectivity for mobile ad hoc network: a survey based study", Wireless Networks, vol. 21, no. 7, (2015), pp. 2369-2394.

[3] A. Boukerche, B. Turgut, N. Aydin, M. Z. Ahmad, L. Boloni and D. Turgut, "Routing protocols in $a d$ hoc networks: A survey”, Computer Networks, vol. 55, no. 13, (2011), pp. 3032-3080.

[4] M. Fekri and K. Shrikant, "A survey of integrating IP mobility protocols and mobile ad hoc networks", IEEE Communications Surveys \& Tutorials, vol. 9, no. 1, (2007), pp. 14-30.

[5] P. M. Ruiz and A. F. G. Skarmeta, "Maximal source coverage adaptive gateway discovery for hybrid $a d$ hoc networks", International Conference on Ad-Hoc Networks and Wireless, Springer Berlin Heidelberg, (2004), pp. 28-41.

[6] U. Javaid, T. Rasheed, D. E. Meddour and T. Ahmed, "Adaptive distributed gateway discovery in hybrid wireless networks", In IEEE Wireless Communications and Networking Conference, (2008), pp. 2735-2740.

[7] S. Bin, K. Haiyan and H. Zhonggong, "Adaptive mechanisms to enhance Internet connectivity for mobile ad hoc networks", In International Conference on Wireless Communications, Networking and Mobile Computing, (2006), pp. 1-4.

[8] M. Alessandrini, G. Biagetti, P. Crippa, L. Falaschetti, S. Orcioni and C. Turchetti, "Optimizing linear routing in the ToLHnet protocol to improve performance over long RS-485 buses", EURASIP Journal on Embedded Systems, vol. 2017, no. 1, (2016), p. 7.

[9] G. Biagetti, P. Crippa, A. Curzi, S. Orcioni and C. Turchetti, "TOLHNET: A low-complexity protocol for mixed wired and wireless low-rate control networks", In: Proceedings of the 6th European Embedded Design in Education and Research Conference (EDERC), Milan, Italy, (2014), pp. 177-181.

[10] G. B. Guarese, F. G. Sieben, T. Webber, M. R. Dillenburg and C. Marcon, "Exploiting modbus protocol in wired and wireless multilevel communication architecture", Brazilian Symposium on Computing System Engineering (SBESC), (2012), pp. 13-18. 
International Journal of Future Generation Communication and Networking Vol. 10, No. 1 (2017) 